\title{
Synthesis Characterization and Catalytic Evaluation of Mesoporous Fe-MCM-41 and Si-MCM-41 Materials
}

\author{
N.Srividhya \\ (Department of Chemistry, PSNA College of Engineering And Technology, Dindigul 624622, India)
}

\begin{abstract}
Environmental concern associated with safe handling disposal of corrosive mineral acids such as $H F$ have encouraged the development of safer and non waste producing alternative for application in catalysis. In this sense, mesoporous materials attracted much attention of catalysis to transform big molecules into its useful products. In the present study we have synthesized, mesoporous Fe-MCM-4land Si-MCM-41 molecular sieves. As synthesized and calcined materials were characterized by XRD and FTIR spectroscopy for the crystallinity and its structural evaluation.

The catalytic activity of Fe-MCM-4land Si-MCM-41 molecular sieves tested for the tertiary butylation of pcresol using tertiary butyl methyl ether. The reaction products are tertiary butyl p-cresol, di-tertiary butyl pcresol and ether identified by Gas chromatography and conformed by Mass spectroscopy techniques.
\end{abstract}

Key words: catalyst mesoporous, Fe/Si-MCM-41

\section{Introduction}

Porous materials act as adsorbents such as active carbons, silica gels, alumina gels and a number of alumina silicates are used directly as catalyst or as support for depositing catalytically-active mixture The progress has been made in a variety of disciplines, including inorganic, materials chemistry, crystallography and petro chemistry [1,2] Zeolites are the examples for micro porous materials having micro porous structure. They are basically hydrated alumina -silicates minerals with an open structure that can accommodate a wide variety of cations such as $\mathrm{Na}^{+}, \mathrm{K}^{+}, \mathrm{Ca}^{2+}, \mathrm{Mg}^{2+}$ and others. The pore sizes are roughly between $10 \mathrm{~A}^{0}$ in diameter. They have the ability to act as catalysts for chemical reactions which take place within the internal cavities. The zeo-type materials (micro porous) can expand their porous to mesoporous range using traditional zeolite synthesis method. The molecular sieves of mesoporous materials represent a new class of inorganic materials first recognized by scientist from Mobil coporation. [3]

\subsection{Synthesis Mechanism}

Procedures used for the preparation of mesoporous materials are similar to those utilized in the synthesis of zeolites here surfactant are used as template instead of inorganic cation or base. Mesoporous material can be prepared under room temperature or mild hydrothermal at $150 \mathrm{c}$ in the presence of anionic or neutral or cationic surfactants either in acidic and base condition. Surfactant chemistry is key to the information of mesoporous materials. Inorganic species also play an important role in organization (electrostatic, vander waal, hydrogen bonding the inorganic species and surfactant molecules which determines the morphology and pore geometry of the resulting mesophase. In order to understand a reaction condition for obtaining a particular mesoporous structure, it is useful to look at surfactant molecules in aqueous solution.

\subsection{Behaviour of surfactants in solution}

In aqueous solution, surfactant molecules exist as very active components with variables structure with increasing concentration. At low concentration, surfactant molecules aggregate together to form micelles, which results in decreases in entropy of the solution.The driving force for the aggregation of surfactant molecule to micelles is to minimize repulsive interaction between their hydrophobic tail and water, so the micelles will becomes in either spherical or rod shaped. The initial concentration at which the surfactant molecules begin to aggregate into micelles is called Critical Micelle Concentration (CMC).

As the surfactant concentration increases further hexagonal close packed array of micelle rod appears, producing hexagonal phase, (such as those that lead to formation of MCM-41). Next step the surfactant concentration increases, the formation of lamellar phase but not always preceded, by the formation of cubic phase.

Micelle formation doesn't only depend on its concentration, but also an nature of surfactant molecules and their environment, length of surfactant hydrophobic alkyl carbon chain, the nature of the surfactant, hydrophilic head group, and the properties of counter ion, ${ }_{\mathrm{p}} \mathrm{H}$, temperature, ionic, strength, and the presence of the other additive do pant, CMC decrease with increase of the chain length of the surfactant, CMC increase with increasing radius, ${ }_{\mathrm{p}} \mathrm{H}$, temperature. A number of mechanisms have been suggested for the formation of 
mesoporous molecules sieves those methods can be classified into two general methods used to prepare mesoporous molecular sieves.

1. Assembly of dissolved inorganic species around surfactant arrays via

liquid crystal templating (LCT) mechanisms.

2. Intercalation of surfactant ions into layered silicates via a folded sheet mechanism (FSM).

\subsection{Liquid crystal t (LCT) mechanisms}

This mechanism first proposed by researchers at the Mobil Corporation for the synthesis of M41S materials, is based on similarities between liquid crystalline surfactant assemblies and the resulting mesoporous solid product. Some of the LCT based formation models have suggested,

1. The puckering layered model.

2. Silicate rod assembly model.

3. Co-operation charge density matching model.

A common feature of all the models is that surfactant in solution conduct the ordering of the material, but the type of interaction between the surfactant and inorganic species are different. Four different reaction pathways which can be explained by this type of mechanism.

The interaction between anionic (negative charged), inorganic species $\left(\mathrm{I}^{+}\right)$and cationic (positively charged ), quaternary ammonium surfactant $\left(\mathrm{S}^{-}\right)$categorized as $\mathrm{S}^{+} \mathrm{I}^{+}$interaction.

Fig 1 General Liquid Crystal Templating (LCT) Mechanism

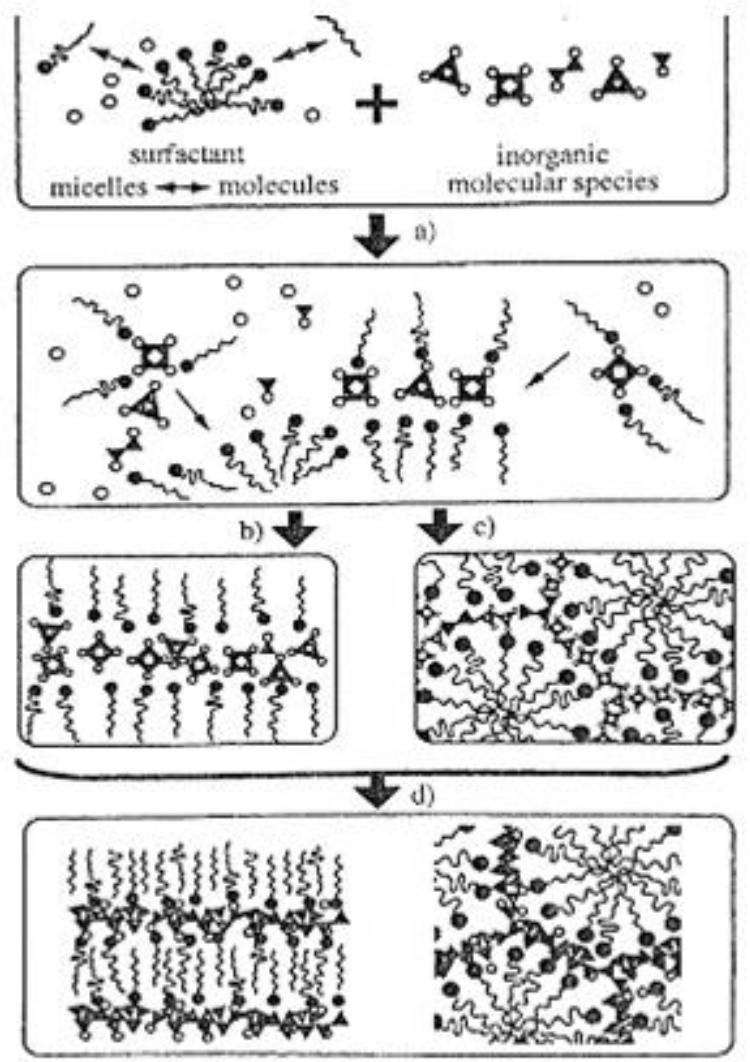

The $\left(\mathrm{S}^{+} \mathbf{I}^{-}\right)$route :

In this case, cationic surfactants $\left(\mathrm{S}^{+)}\right.$are used as structure directors for anionic inorganic species $\left(\mathrm{I}^{-}\right)$. This is recognized as the pathway for the synthesis of MCM mesoporous materials.

The $\left(\mathrm{SI}^{+}\right)$route :

In this pathway anionic surfactant $\left(\mathrm{S}^{-}\right)$interact with cationic inorganic species $\left(\mathrm{I}^{+}\right)$

The $\left(\mathbf{S}^{+} \mathbf{X}^{-} \mathbf{I}^{+}\right)$route:

Here both the surfactant and the inorganic species are cationic .This pathways is mediated by negatively charged counter ions $\left(\mathrm{X}^{-}=\mathrm{CI}^{-}, \mathrm{Br}^{-}\right)$etc. 
The $\left(\mathbf{S}^{-} \mathbf{M}^{+} \mathbf{I}^{-}\right)$route :

In this case both the surfactant and the inorganic species are anionic and positively charged counter ions $\left(\mathrm{M}^{+}=\mathrm{Na}^{+}, \mathrm{K}^{+}\right)$etc., mediate the structure formation. The other types of the surfactant inorganic interactions such as these involving neutral surfactant as structure director (templates).

1. $\mathrm{S}^{0} \mathrm{I}^{0}$ where both the surfactant $\left(\mathrm{S}^{0}\right)$ and the inorganic species $\left(\mathrm{I}^{0}\right)$ are neutral. The surfactant - inorganic interaction is due to hydrogen bonding.

2. $\quad \mathrm{N}^{0} \mathrm{I}^{0}$, where both the templates, nonionic polyethylene oxide $\left(\mathrm{N}^{0}\right)$ and inorganic species $\left(\mathrm{I}^{0}\right)$ are neutral. The organic-inorganic interaction is due to hydrogen bonding.

Fig 2 Mechanism of the mesoporous material formation (hexagonal, MCM-41)

\section{LCT Liquid Crystal Templating}

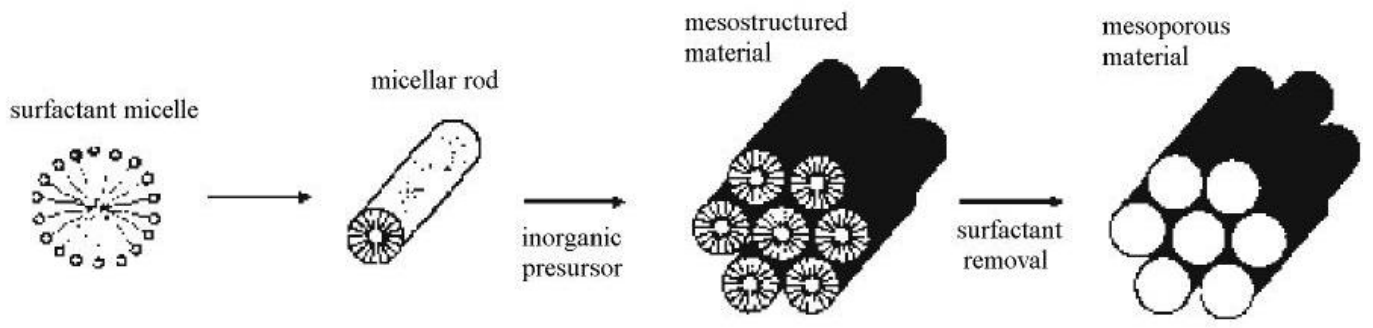

\subsection{Folded Sheet Mechanism}

This mechanism is therefore based on the intercalation of ammonium surfactant in kanemite, a type of hydrated sodium polysilicate composed of single layered silica sheets. After the surfactant are ion exchanged into the layered structure, the silicate sheets are thought to fold around the surfactant and condense into a hexagonal mesostrucure.

Since siliceous mesoporous materials have not enough intrinsic acidity to be catalytic activity is, however, enchanced by modification of the siliceous framework by other elements like trivalent cations A1, B, $\mathrm{Ga}$, Fe substitute for silicon in the walls of the mesoporous silica [4] the framework possesses negative charges that can be compensated by protons and solids can be used in acidic reactions. When other cations like Ti, V, $\mathrm{Sn}, \mathrm{Zr}$ are introduced, the electro neutrality is maintained and the corresponding mesoporous materials are used rather in specific reaction like in redox catalysis.

\subsection{APPLICATION OF MESOPOROUS MCM-41 MOLECULAR SIEVES}

A variety of solid acids besides zeolites have been tested as alkylation catalysts. The Friedel-Crafts alkylation of electron rich aromatic compounds. In addition, the rather large pore openings of mesoporous materials offer the advantage of effective diffusion of bulky substances as they are often found in fine chemical synthesis. One of the earliest examples is that of a Friedel-Crafts alkylation with mesoporous alumino silicates. The catalytic activity in the alkylation of unsubstituted phenol with methanol over A1-MCM-41, which had been modified by grafting of an alumina-multilayer was up to 2.3 times higher than that of a reference alumina catalyst.[5] In the Friedel crafts alkylation of benzene with benzyl chloride in liquid phase, Ga impregnated MCM-41 [6] exhibited higher activity than Ga substituted MCM-41. In series consisting of Fe-impregnated MCM-41, A1-MCM-41, La-MCM-41and Fe-USY zeolite in the same reaction, the Fe-impregnated MCM-41 showed the highest activity. [7]

In this work, we have synthesized mesoporous Fe-MCM-41, Si-MCM-41 catalyst with various $\mathrm{Si} / \mathrm{M}$ $(\mathrm{M}=\mathrm{Si}, \mathrm{Fe})$ ration and phosphotungstic acid impregnated $\mathrm{Si}-\mathrm{MCM}-41$ catalyst to study the performance of SiMCM-41 and Fe-MCM-41 and PWA/Si-MCM-41 catalyst in the tertiary butylation of p-cresol with tertbutylmethylether (TBME).

\subsection{Materials}

\section{Materials And Methods}

Chemicals such as sodium meta silicate $(\mathrm{CDH})$ cetyltrimethylammoniumbromide $(\mathrm{CTAB})$, aluminum sulphate, ferrous sulphate used for synthesis of mesoporous molecular sieves. sulphuric acid was used to adjust the $\mathrm{pH}$ medium. Chemicals such as P-cresol, Tertiarybutylmethylether, $\mathrm{KBr}$, Silicone oil, acetone, methanol was of analar grade. The glass ware items used in all the experiments and the fabricated glass reactors are made of borosil. 
Synthesis Characterization and Catalytic Evaluation of Mesoporous Fe-Mcm-41 and .....

\subsection{Analysis}

XRD analysis of hydrothermally synthesized and impregnated materials was performed on a Philips Xpert diffractometer equipped with solid-state detector using $\mathrm{CuK} \alpha$ radiation. The samples were scanned between 0.5 and $8.5(2 \theta)$ in steps of $0.02^{\ominus}$ with the counting time of $5 \mathrm{sec}$ at each point.

The catalysts were characterized by FTIR spectroscopy in a Broler IFS66V spectrometer using potassium bromide $(\mathrm{KBr})$ pellet technique. About $20 \mathrm{mg}$ of the sample was ground with around $200 \mathrm{mg}$ of spectral grade of potassium bromide to form a mixture, which was then made into pellet using a hydraulic press. The mixture was pressed at $500 \mathrm{Kgcm}^{-2}$ into a thin wafer. The pellet was then to record infrared spectra at room temperature in the region of $4000-400 \mathrm{~cm}^{-1}$

Quantitiative analysis of the liquid products was carried out by gas chromatography Perkin Elmer (Claris 500 model) equipped with Flame Ionization detector and elite $5 \mathrm{~ms}$ capillary column. The products were further identified by Shimadzu GC-MS-QP1000EX gas chromatograph mass spectrometer. Each component of the products mixture was identified from its $\mathrm{m} / \mathrm{e}$ value and the fragmentation pattern.

\subsection{Synthesis of Fe/Si-Mcm-41 Molecular Sieves}

A series of mesoporous materials under hydrothermal conditions have been synthesized. Sodium metasilicate and ferrous sulphate were used as the sources for iron and silicon respectively. Cetyltrimethylammoniumbromide (CTAB) was used as the structure directing template. In a typical synthesis an appropriate amount of sodium metal silicate and aluminium sulphate is weighted and it is dissolved in required amount of distill water in long neck $500 \mathrm{ml}$ beaker and it is kept for stirring using mechanical stirrer for one hour at room temperature. After one hour dilute sulphuric acid is added drop by drop using pipette until the silica gel is obtained and it is allowed to stir for two hours until the homogeneous solution is obtained. After $2 \mathrm{~h}$ the known amount of cetrimide solution (surfactant) is added to get homogeneous solution, it is allowed to stir for $2 \mathrm{~h}$. This solution is poured in the autoclave and it is kept at oven under the temperature of $150^{\circ} \mathrm{C}$ for $24 \mathrm{~h}$. This solution is filtered using suction pump with ordinary filter paper. The filtered substance is dried at oven under temperature of $100^{\circ} \mathrm{C}$ for one hour. After the substance is dried well it is crushed and powered well using motor and pestle. That powered substance is kept for calcinations under the temperature of $550^{\circ} \mathrm{C}$. The calcined sample is crushed and powered well using mortar and pestle.

\subsection{Preparation of PWA Impregnated Catalysts}

Using phospotungstic acid (PWA) various weight ratio of PWA impregnated Si-MCM-41 catalyst was synthesized by incipient wetness method.

\subsection{Experimental Procedure For Liquid Phase Alkylation Reaction}

The alkylation of p-cresol with ter-butyl methyl ether (TBME) was carried out in a liquid phase reactor prior to the reaction the catalysts were activated in pure dry air at $55^{\circ} \mathrm{C}$ for $5 \mathrm{~h}$. The reaction was carried out in a two necked round bottom flask. The molar rations of reactant and alkylation agent were 1:1, 1:2, 1:3, and $2: 1$.The reaction was performed at $120^{\circ} \mathrm{C}$ for $8-12 \mathrm{hrs}$. The temperature of the reaction is controlled by dimmer stat reaction samples collected with the help of glass syringes at the intervals of $2 \mathrm{~h}$. No significant p-cresol conversion was observed when the reaction was carried out without catalyst indicating that there is no thermal effect on conversion.

\subsection{X-Ray diffraction analysis}

\section{Results and Discussion}

The X-ray diffraction patterns of calcined Fe-MCM-41(50) and Si-MCM-41 (50) samples are presented in the fig 3.1.1, 3.1.2 respectively. The XRD pattern shows a prominent peak corresponds to (100) reflection followed by much weak but clearly present peaks corresponding to (110), (200), (210) reflections. The calcined samples showed a d(100), (110), (210) spacing of 2.1, 3.25, and 3.8 respectively. X-ray diffraction of Fe-MCM41 samples present a typical 3 pattern with a very strong peak at a low angle (100) and other 2 peaks corresponding to 110 and 200 planes. All shows that these crystals do have a hexagonal symmetry.

\subsection{FTIR Spectroscopy}

The FTIR spectrums in the regions $400-4000 \mathrm{~cm}^{-1}$ of uncalcined and calcined Si-MCM-41 catalyst are given in the fig.3.2.1, 3.2.2 respectively. The presence of isolated surface silonals hydrogen bonded, hydroxyl groups, and amorphous structures of the wall are evidenced form the IR spectrum of the calcined Si-MCM-41 sample. The band at $1023 \mathrm{~cm}-1$ is assigned to the symmetry vas ( $\mathrm{Si}-\mathrm{O}-\mathrm{Si}$ ) vibrations and the band at $795 \mathrm{~cm}^{-1}$ is assigned to symmetric vibrations of Si-MCM-41. In the hydroxyl region $\left(3600-3200 \mathrm{~cm}^{-1}\right)$ a broad band is seen assigned to the silonal group inside the channels of Si-MCM-41. 
FTIR spectra of Fe-MCM-41 and Si-MCM-41 synthesized and calcined materials are visualized in the figure 3.2.1, 3.2.3 respectively. It show some important differences in the intensity of surface silanol groups. The decrease intensity of the silanol group after Fe and Si grafting shows the active participation of the surface silanols in bond formation with the iron and silicon precursor. The vibrational frequency of $984.2 \mathrm{~cm}^{-1}$ attributed to $\mathrm{Si}-\mathrm{OH}$ vibrations.

\subsection{Catalytic Studies}

The ter-butylation of p-cresol by ter-butylmethylether over various mesoporous Fe- MCM-41 and Si-MCM-41 catalyst have been carried out in the liquid phase reactor. The main products are TBC, DBPC, and ether. The reaction shown in the fig.1.8 This reaction is an example of electrophilic substitution where tert-butyl cation formed by tert-butyl methyl ether substituted on the aromatic ring to $\mathrm{C}$-alkylated products and also small amounts of O-alkylated products.

\section{Conclusion}

Iron and silicon containing mesoporous MCM-41 molecular sieves were synthesized through hydrothermal route, physico-chemical characterizations X-ray diffractions techniques and FTIR spectroscopy reveals that incorporation of iron and silicon elements into the frameworks of mesoporous networks. Catalytic measurements showed that iron and silicon containing mesoporous molecular sieves are capable of performing tertiary butylation reaction of polar molecules like p-cresol. Although the present date are not sufficient to establish the detailed mechanism of tertiary butylation of p-cresol reaction. On the basis of the results obtained it is believed that the alkylation of p-cresol with methyl tertiary butyl ether occurs in a good manner. Hence, it is concluded from this study that silicon and iron containing mesoporous MCM-41 molecular sieves could be an active catalyst for various alkylation reactions.

[1]. Smith,J V. Chem.Rev.(1998),88,149-182.

[2]. A.Corna, V. Forne's M.T. Navarro, J. Pe'rez-Paritente, J.Catal.148, (1994),569.

[3]. C.T. Kresge, M.E. Leonowicz, W.J. Roth, J.C. Vartuli, J.S. Beck, Nature 359, (1992), 710

[4]. M. Baltes, A. Kytokivi, B.M. Weckhuysen, R.A. Schoonheydt, P. Van Der Voort, E.F. Vansant,

[5]. J. Phys. Chem. B 105, (2001), 6211.

[6]. M.V. Landau, E.Dafa, M.L. Kaliya, T.Sen, M.Herskowit, Micropor. Mesopor. Mater. 49, (2001), 65.

[7]. K. Okumura, K.Nishigaki, M. Niwa, Micropor. Mesopor. Mater. 44-45,(2001), 509.

[8]. N. He, S.Bao, Q. Xu, Appl. Catal. A: Gen 169, (1998), 29.

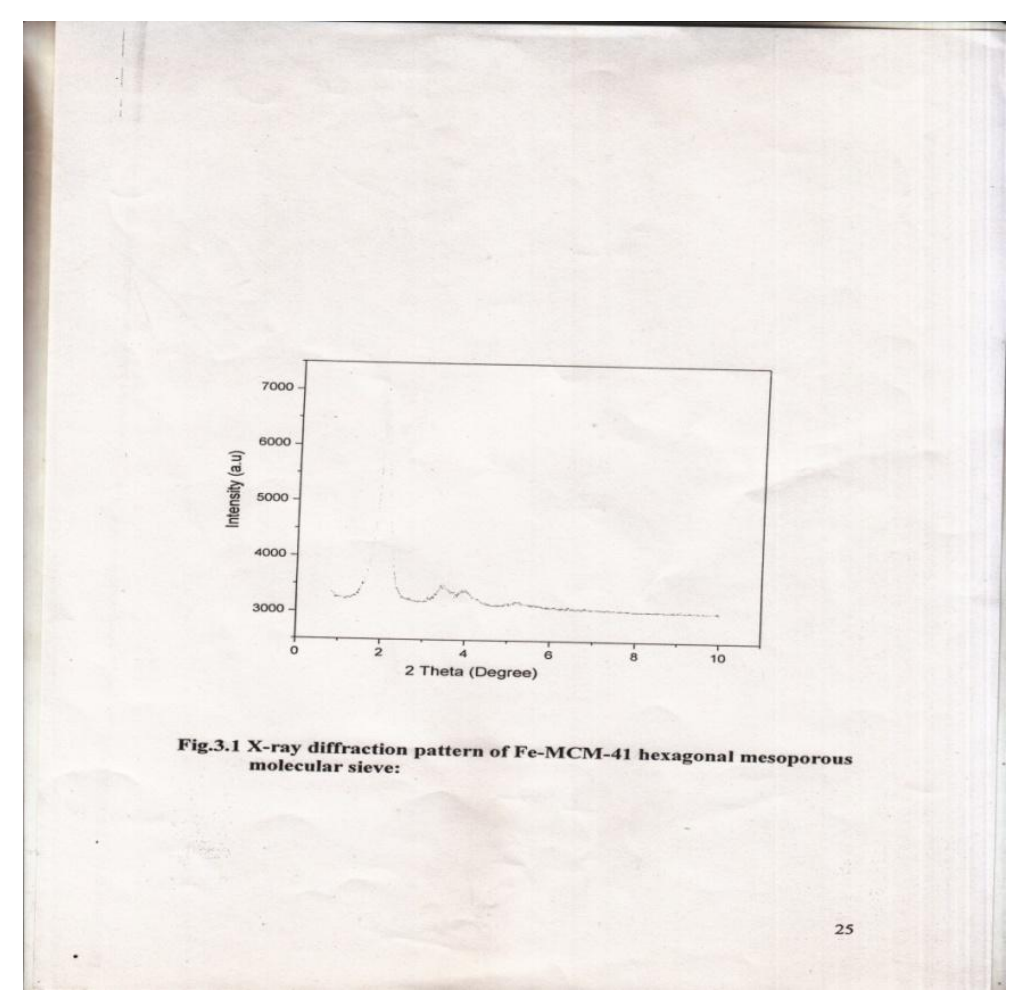

Fig. 3.1.1 


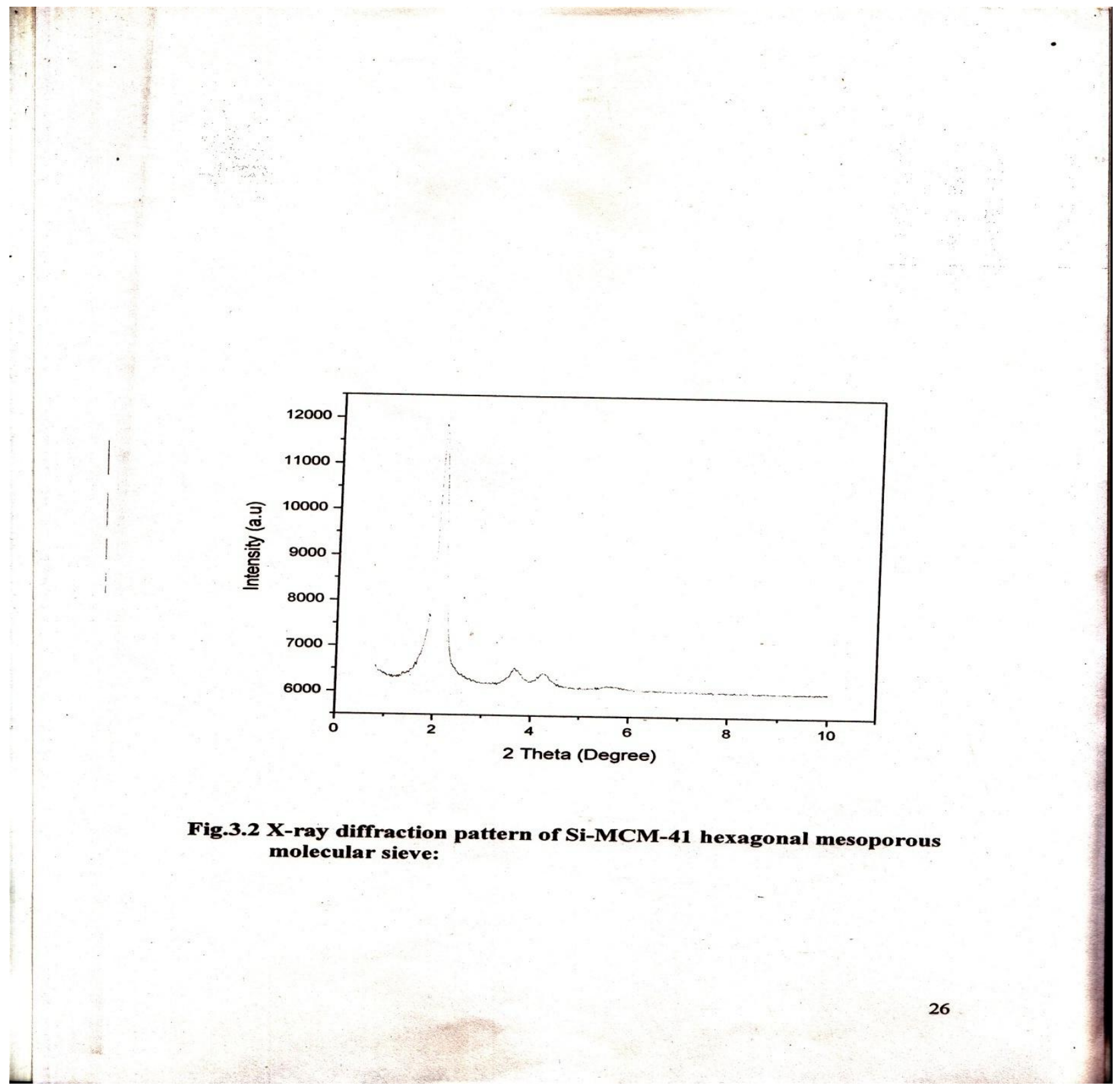

Fig. 3.1.2 
$\sqrt{2}$
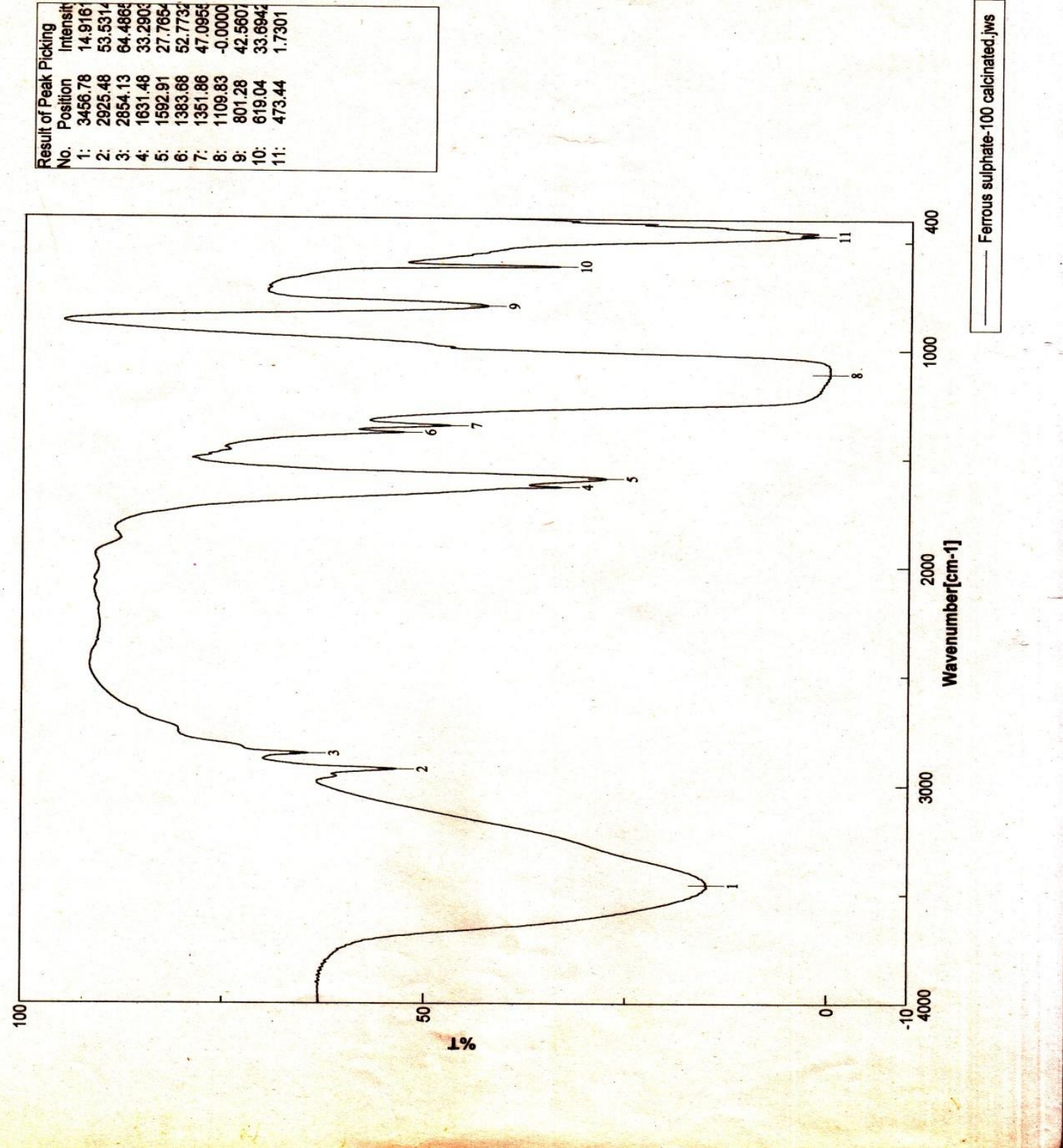

Fig 3.2 .1 


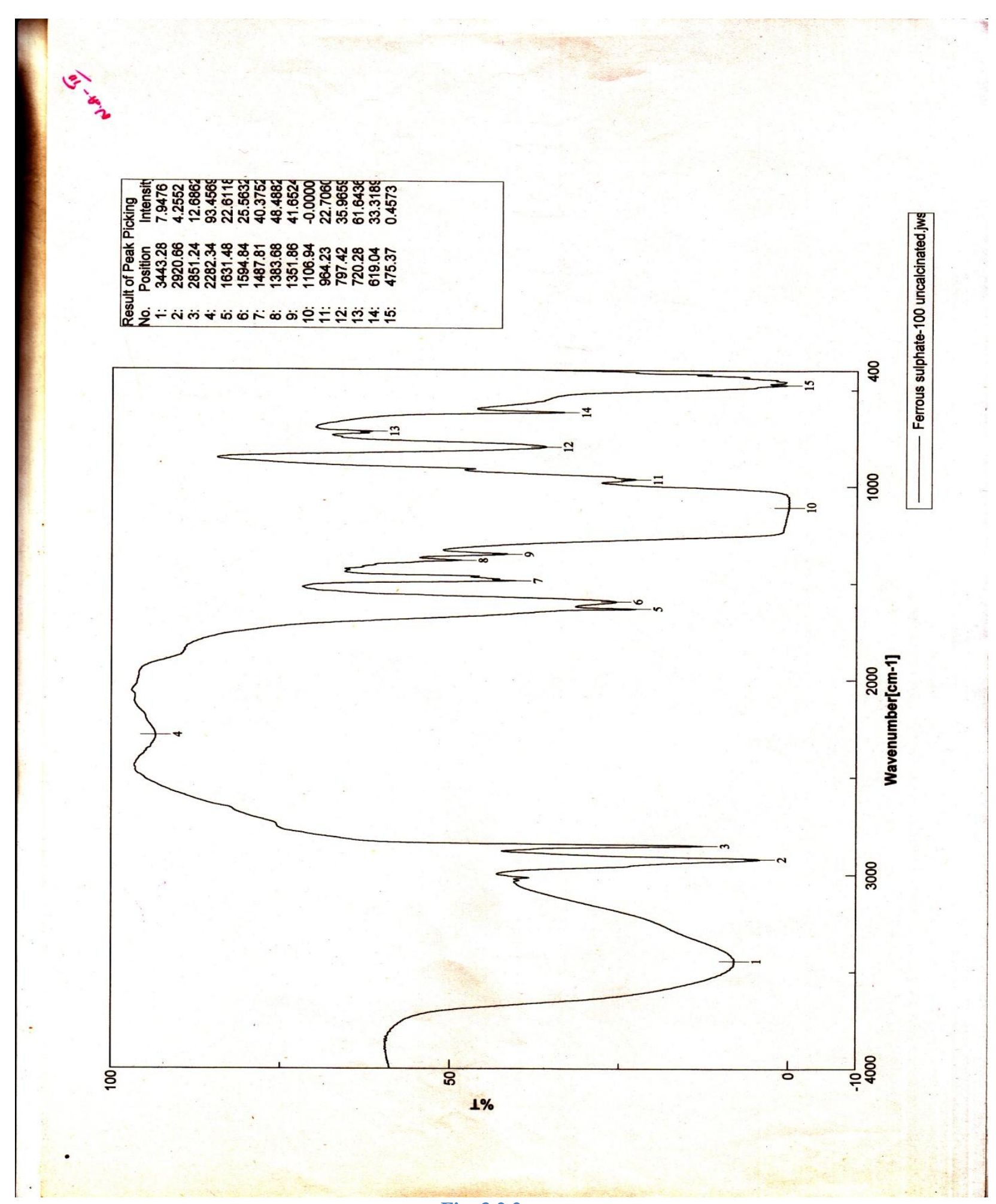

Fig. 3.2.2 
(ब)
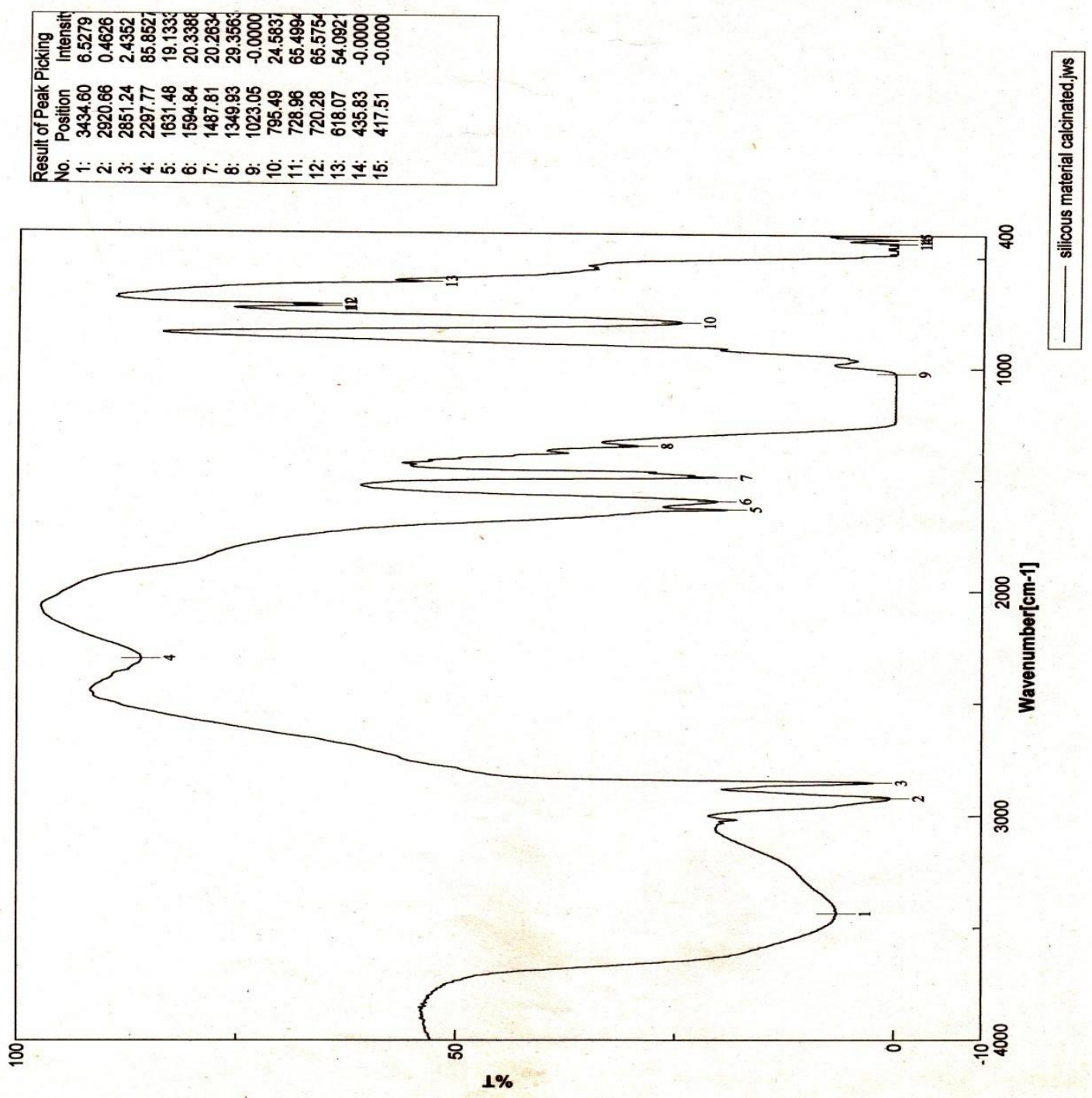

Fig. 3.2.3 